\title{
Migración de talentos COMO ESTRATEgia DE DESARROLlO: MÉXICO-JaPÓN
}

\author{
Alejandro Méndez Rodríguez ${ }^{1}$
}

Fecha de recepción: 18 de enero de 2017. Fecha de aceptación: 24 de marzo de 2017.

\begin{abstract}
RESUMEN
La migración internacional de recursos humanos calificados es uno de los pilares de la gobernanza de la inmigración. Las políticas migratorias establecen los instrumentos de organización y gestión de la misma con la finalidad de atraer trabajadores calificados y estudiantes internacionales en el contexto de la competitividad global. El principal componente que está influyendo en la construcción del flujo migratorio internacional hacia Japón, es el mercado transnacional laboral de recursos calificados y de los mecanismos de su formación. El objetivo de este artículo consiste en señalar los factores socioeconómicos que configuran, impulsan y dan contexto a la movilidad de trabajadores calificados. Y la interrogante principal sería: ¿Cuáles son las características de los principales factores que están moldeando el flujo migratorio entre México y Japón?
\end{abstract}

Palabras clave: migración calificada, movilidad geográfica, recursos humanos, factores socioeconómicos, mercados de trabajo, política migratoria.

Clasificación JEL: F22, O15, R23.

\section{Talent Migration as a Development STRATEgY: MeXico-Japan}

\begin{abstract}
The international migration of qualified human resources is one of the pillars of immigration governance. Immigration policies set instruments for organizing and managing these human resources to attract skilled workers and international students in a context of global competitiveness. The main component influencing the dynamics of international migration flows to Japan is the transnational labor market of skilled resources, as well as the mechanisms that shape it. The objective of this paper is to describe the socioeconomic factors that shape, drive, and lend context to the mobility of skilled workers. The main question to answer would be: What are the characteristics of the main factors shaping migration flows between Mexico and Japan?
\end{abstract}

Key Words: Skilled migration, geographic mobility, human resources, socioeconomic factors, labor markets, migration policy.

1

Instituto de Investigaciones Económicas de la Universidad Nacional Autónoma de México. Correo electrónico: menrod@unam.mx 


\section{MIGRATION DE TALENTS COMME STRATÉGIE DU DÉVELOPPEMENT: MEXIQUE- JAPON \\ Résumé}

La migration internationale de ressources humaines qualifiées est l'un des fondements mêmes de la gouvernance de l'immigration. Dans le contexte de la compétitivité globale, son organisation et sa gestion sont établies par les politiques migratoires afin d'attirer de personnels qualifiés et des étudiants internationaux. La composante la plus importante qui influe sur la construction du flux migratoire international vers le Japon est le marché du travail transnational de ressources qualifiées et les mécanismes de sa formation. Le but de cet article consiste à identifier les facteurs socio-économiques qui configurent, stimulent et donnent le contexte à la mobilité de personnels qualifiés. Notre point d'intérêt principal est : identifier quelles sont les caractéristiques des principaux facteurs qui modèlent le flux migratoire entre le Mexique et le Japon.

Mots clés: migration qualifiée, mobilité géographique, ressources humaines, facteurs socio-économiques, marchés du travail, politique migratoire.

\section{MIGRAÇÁO DE TALENTOS COMO ESTRATÉGIA DE DESENVOLVIMENTO: MÉXICO- JAPÁO \\ Resumo}

A migração internacional de recursos humanos qualificados é um dos pilares da governança da imigração. As políticas de migração estabelecem os instrumentos organização e gestão que têm como objetivo atrair trabalhadores qualificados e estudantes internacionais no contexto de competitividade global. O componente principal que influencia na construção do fluxo migratório internacional para o Japão é o mercado de trabalho transnacional para recursos qualificados e dos mecanismos de sua formação. O objetivo deste artigo é apontar os fatores socioeconômicos que moldam, fomentam e dáo o contexto para a mobilidade de trabalhadores qualificados. E a principal pergunta seria: Quais sấo as características dos principais fatores que estáo moldando o fluxo migratório entre México e Japão?

Palavras-chave: migração de trabalhadores qualificados, mobilidade geográfica, recursos humanos, fatores socioeconômicos, mercados de trabalho, política migratória.

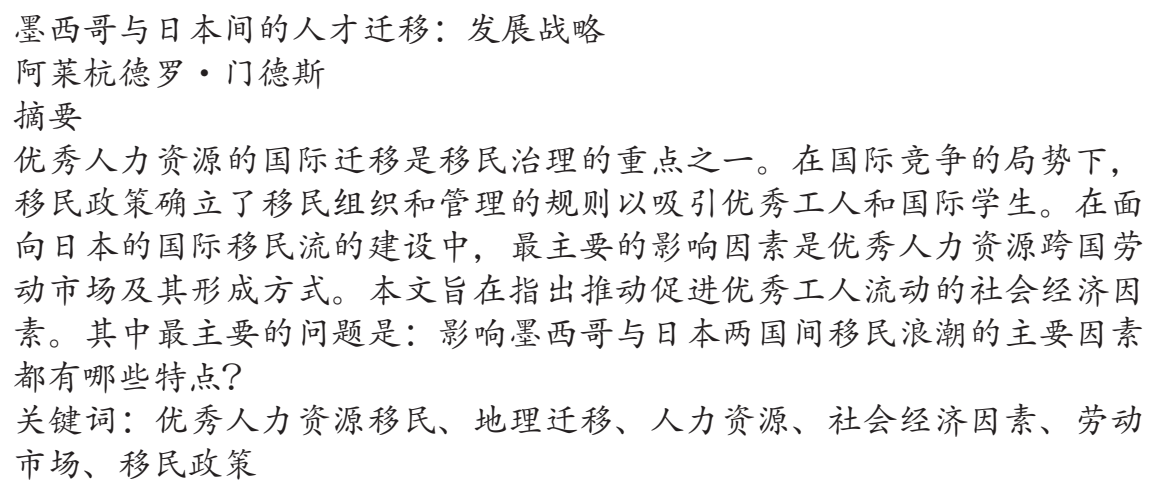




\section{INTRODUCCIÓN ${ }^{2}$}

El crecimiento económico registrado en Japón en la segunda parte del siglo $\mathrm{xx}$, se fincó en la innovación sin importantes flujos de inmigración de mano de obra extranjera. A partir de la década de los ochenta, el desarrollo económico requirió de la inmigración de trabajadores, preferentemente de personas con vínculos de origen japonés, denominada población nikkei. En el presente siglo, en Japón se amplían notablemente los esquemas migratorios a los inmigrantes calificados, tanto profesionistas como estudiantes.

La política de inmigración japonesa está vinculada al mercado laboral y al sistema educativo, lo que se demuestra con el flujo migratorio, el que se encuentra fuertemente marcado por la internacionalización de la educación y el modo de producción de la economía global. Cabe destacar tres factores que soportan el flujo migratorio México-Japón: la presencia de las comunidades nikkei y las ventajas laborales y educativas para los recursos humanos que ofrece Japón. A corto plazo, con base en la tendencia estadística se estima una ampliación del flujo migratorio.

La creación de espacios para la migración calificada en Japón está guiada por el principio de una selección de personas que favorezcan el desarrollo económico, social y cultural. Se pone el énfasis en la población con estudios. Se evita el ingreso de personas de baja calificación. Se tiene la visión de que un segmento de la sociedad japonesa seguirá realizando las actividades de baja calificación. Por ejemplo, el gremio de taxistas es eminentemente japonés. Se protege el mercado laboral y se abre en los renglones que se necesitan.

El presente artículo ofrece una visión estructural del fenómeno de la migración internacional de recursos humanos calificados. La migración es un producto social resultado de factores macros como el funcionamiento del mercado laboral transnacional, así como, de elementos mezzo como las políticas de inmigración de los Estados-nación. Además, factores micro como son los aspectos subjetivos y valorativos del trabajador internacional, en donde interviene la variable de rechazar empleos inferiores y, por ende, buscar condiciones de vida mejores allende de las fronteras nacionales. Este artículo apunta los elementos macro que están influyendo en la construcción del flujo migratorio internacional hacia Japón, centrándose en los rasgos generales del mercado transnacional laboral de recursos calificados y de los mecanismos

\footnotetext{
El autor agradece a la Dirección General de Asuntos del Personal Académico de la unam, el apoyo brindado para realizar una estancia sabática en Tokio, Japón. Agradece también a Manuel Méndez Astudillo, el apoyo en la recopilación y traducción de la información estadística.
} 
de su formación, todo ello, englobado en los instrumentos de organización y gestión de la migración internacional.

Asimismo, el objetivo principal del texto consiste en señalar preferentemente los factores japoneses que configuran, impulsan y dan contexto a la movilidad de trabajadores calificados. La pregunta de investigación es: ¿Cuáles son las características de los principales factores que están construyendo el flujo migratorio México-Japón?

Los vínculos entre México y Japón están presentes desde hace 400 años (Girón, Vargas \& Uscanga, 2015). La Misión Hasekura con destino a Nueva España que data del año de 1624 se "ha convertido en un referente fundamental desde inicios del siglo xx hasta la actualidad para expresar los añejos lazos de amistad entre México y Japón” (Uscanga, 2015, p. 45). Actualmente, la presencia japonesa en México es visiblemente notable desde el ángulo de la inversión extranjera directa, que se concentra en la región centro de México. Empresas y recursos financieros y humanos de origen japonés avivan el panorama económico de esa región geográfica. Naturalmente, este hecho constituye un factor dinamizador del flujo migratorio contemporáneo.

Ahora bien, qué elementos emergen en Japón para construir el flujo migratorio desde la perspectiva de las estrategias de desarrollo. Históricamente, la movilidad de población ha sido considerada como una estrategia de desarrollo de las sociedades, en el caso particular en Japón, su historia migratoria muestra el estrecho vínculo entre migración y desarrollo; por ejemplo, el flujo migratorio de Japón a Brasil en el siglo xx (Lesser, 2003). Desde la óptica de los países expulsores de población, la migración internacional como estrategia de desarrollo ha sido documentada ampliamente a través del análisis de las remesas. Algunos gobiernos plantean explícitamente a la migración como una estrategia de desarrollo nacional, por ejemplo, Filipinas. En el caso de México se reconoce a la migración como detonador de crecimiento a partir de la transferencia de remesas.

\section{MOVILIDAD, MIGRACIÓN Y FLUJOS}

La movilidad de personas en busca de recursos materiales para el sustento es un proceso inmerso en la historia económica capitalista, tal como lo documenta Saskia Sassen (2014) en el estudio sobre 200 años de migración en Europa. El fenómeno de la migración es una modalidad que adquiere la movilidad en el esquema de los sistemas políticos de los Estados-nación y que se expresa bajo diversos matices, según los espacios y tiempos específicos. De ahí, la distinción 
analítica entre movilidad, migración y flujos, sin embargo, estos términos están embebidos mutuamente. Por eso, la movilidad geográfica de los recursos humanos capacitados ha sido analizada bajo la mirada de diversos conceptos de los estudios migratorios (Bartram, Poros \& Monforte, 2014): diáspora, circulación de talentos, migración de retorno, trabajadores invitados y otros.

La migración internacional como movilidad de capital humano es un elemento clave en el bienestar de los Estados-nación receptores de ese flujo, y de los países expulsores. Desde esta óptica, en general la política de migración está dirigida a contribuir al desarrollo y a la reducción de la pobreza de los países tanto receptores como expulsores. El flujo migratorio se ubica bajo los principios de la gobernanza de las migraciones, que establece los mecanismos de gestión y control de la migración. Actualmente, el eje de esos mecanismos descansa en el principio de selectividad de los inmigrantes, para ello, se establecen diversos criterios de admisión de migrantes. Esta situación ha hecho que las políticas de migración adquieren el cariz de restrictivas y fuertemente selectivas.

Este artículo se centra básicamente en la categoría, los migrantes económicos en los grupos de trabajadores calificados y de estudiantes internacionales. Ambos actores forman parte de la migración de talentos. Al respecto, las políticas migratorias han puesto el acento especialmente en el proceso de aceptación y admisión de la población calificada y de estudiantes. Este proceso está construido históricamente por los Estados-nación que dependen de las condiciones estructurales y coyunturales de la economía. Por eso, se han aplicado diversos criterios de selección de inmigrantes, tales como los recursos económicos, el nivel educativo, los vínculos familiares y las afinidades culturales. Los sistemas selectivos de admisión de migrantes establecen, comúnmente, variables como la edad, escolaridad, dominio del lenguaje oficial del país receptor, así como las habilidades profesionales y la experiencia en el trabajo.

El flujo de migración está acotado y gestionado por parte de los Estadosnación, en ocasiones por los países receptores y por los expulsores, mediante la búsqueda de población calificada. La relevancia de los migrantes calificados en el contexto de los flujos migratorios se ilustra con el hecho de que 67 de 170 países han instrumentado políticas de admisión de personas calificadas. Además, 137 de 145 países han registrado un aumento en el número de migrantes calificados (Zapata-Barrero \& Pasetti, 2015, p. 84).

La migración de recursos humanos calificados ha sido un tema abordado por las ciencias sociales, se han empleado ciertos términos con fines de clasificación que conlleva perspectivas de análisis. Con el paso de los años se han acuñado conceptos descriptivos, como es el caso de fuga de cerebros; mismo 
que, ha transitado por diversos momentos. En los años sesenta del siglo pasado surge haciendo referencia a la movilidad de estudiantes y profesionistas de área de la ciencia y la tecnología. Las investigaciones se centraron en analizar los factores nacionales de expulsión. El segundo es la ganancia de cerebros, ello pone el énfasis en los mecanismos de atracción que ofrecen los sistemas inmigratorios. El tercer momento es la circulación de cerebros, se incorpora la idea de la migración de regreso de los recursos humanos calificados para reintegrarse a la vida económica del país de origen, por ejemplo, se observa que los talentos regresan para emprender y dirigir negocios transnacionales. En cambio, la circularidad o el retorno de estudiantes internacionales menores de edad a sus lugares de origen acarrea diversas dificultades de índole de reingreso (Bazán-Ramirez \& Galván-Zariñana, 2013).

Estos enfoques han considerado a la migración de talentos como el desplazamiento entre dos polos, sin embargo, la mundialización del fenómeno migratorio plantea nuevos retos de investigación, debido a la red de flujos migratorios entre diversos polos. La migración calificada afronta diversos problemas en las sociedades receptoras. En estudios recientes se han establecido las barreras múltiples que enfrentan los migrantes calificados en la integración a la sociedad de destino cuando el idioma oficial no es el materno de los inmigrantes (Sardana, Zhu \& Veen, 2016). Pero también, los migrantes se enfrentan a la discriminación para obtener un trabajo deseado, por lo general, obtienen salarios bajos e insatisfacción laboral, principalmente por los sistemas de acreditación y reconocimiento de estudios. Los migrantes aceptan trabajos inferiores a sus calificaciones, a pesar de ser considerados como "capital humano apropiado" para el desarrollo nacional en el marco de la competitividad global.

El flujo migratorio está marcado por dos hechos sobresalientes: la migración de trabajadores calificados y la movilidad de los estudiantes internacionales, ambos convergen en la ruta de la migración de y por estudios, en donde se privilegia el proceso de inmigración a partir de credenciales educativas. Estos grupos difieren en su trayectoria migratoria. Mientras que los trabajadores calificados obtienen la condición de ciudadanía en pocos años; los estudiantes internacionales se estiman, por ejemplo, que adquieren el estatus de ciudadano después de 16 años de haber ingresado a Estados Unidos (Hawthorne, 2014, p. 6). Otros países, como Canadá instrumenta el principio de Canadian Experience Class para facilitar la retención de los estudiantes internacionales en el año de 2008. 
El proceso de globalización ha acentuado el mercado de exportación de la educación, las instituciones de educación superior ofrecen educación internacional de alta calidad en varios campos como las tecnologías de la información, las ingenierías y en los negocios, entre otras. En el proceso de migración de estudiantes internacionales, se han observado pautas de comportamiento eminentemente comerciales de las instituciones educativas privadas, como el caso de Australia, en donde, los estudiantes generaron anualmente cerca de 18 billones de dólares australianos entre 2001 y 2009. La educación se convirtió en la tercera industria más importante en Australia (Hawthorne, 2014, p. 6).

\section{La migración internacional de capital humano en Japón}

Los gobiernos nacionales reorientan sus estrategias de desarrollo en el marco de la globalización. El discurso de la competitividad internacional domina las líneas de desarrollo nacionales. En términos generales, la estrategia competitiva adoptada por México consistió en desarrollar productos baratos con base en la reducción de los costos laborales; en cambio, otros países como Japón instrumentaron estrategias de economías de escala e innovación tecnológica. De manera particular, la competitividad se aplica, también, en los recursos humanos. Esto se ilustra con el hecho de la formación de recursos humanos para competir en el mundo globalizado realizando tareas de competencia internacional.

Muy probablemente, Japón es el país más altamente desarrollado que alcanzó un crecimiento económico en los ańos sesenta y setenta del siglo xx, sin recurrir a la importación de mano de obra extranjera, ya que fincó su desarrollo en la productividad laboral; lo cual, no significó que no participaran trabajadores extranjeros en el mercado de trabajo japonés. Cabe señalar que antes y después de la Segunda Guerra Mundial, la presencia de coreanos fue notable (se calcula en 2.1 millones). Las oleadas de coreanos data de principios del siglo xx (Weiner, 1994).

Durante el siglo xx, la movilidad de población en Japón tuvo diversos momentos que fueron desde ser considerado como un país expulsor de mano de obra a uno que atrajo mano de obra, principalmente, de los países de la región asiática como: China, Corea del Sur y Vietnam. De acuerdo con Junichi Goto (2008), durante ese siglo, la migración de la población japonesa experimentó cinco grandes momentos de emigración e inmigración. Las cuatro primeras etapas son eminentemente de expulsión de población como estrategia de 
crecimiento económico, y la última fase del momento actual se distingue por ser de atracción de población bajo la tendencia de la mundialización.

Tradicionalmente, se considera en los estudios migratorios que los imperios coloniales crean fuertes puentes para la migración. Sin embargo, en la actualidad debe tomarse en cuenta, también, las actividades económicas transnacionales como creadoras de enlaces de la migración (Sassen, 2014, p. 203). De acuerdo con ello, para el caso de México, destaca la inversión directa japonesa en la zona económica del Bajío como un motor de la movilidad de la población. Ahora bien, ¿cuáles son las características de la política de inmigración de Japón en los últimos años?

Autores como Tsuda y Cornelius (2004) destacan la discrepancia entre la política migratoria oficial y la realidad económica japonesa. Por otro lado, la regulación y control del flujo migratorio establece que está cerrada la puerta para trabajadores extranjeros que no tengan preparación. Entre 1975 y 1985, se crearon 6 millones de empleos en Japón (Sassen, 2014, p. 198). Además, entre los años de 1986 y 1991, el mercado laboral se expandió en 4.4 millones de empleos (Tsuda \& Cornelius, 2004, p. 444). En los años ochenta, Japón se convirtió en la región asiática como un país de atracción de migrantes debido al dinamismo económico y, por supuesto, a las diferencias salariales. El flujo migratorio es resultado, como ya se ha señalado, de diversos factores económicos transnacionales como las inversiones directas de Japón realizadas en Corea, China, Filipinas y Tailandia; además, factores sociopolíticos como la presencia de refugiados, históricamente se encuentran la población de Vietnam. También son pertinentes los factores etnoculturales como lo muestra la comunidad nikkei (población en diversos países que tienen ancestros japoneses). Para el siglo XXI, Japón se trasforma en un país que atrae trabajadores calificados y estudiantes internacionales.

Se ha afirmado que el flujo de capital japonés y la relocalización de la producción de las firmas multinacionales crean oportunidades de empleo para los trabajadores nativos locales, y da a ellos el acceso a recursos y conexiones necesarias para migrar a Japón (Sassen, 1991, p. 32). A pesar de que en Japón no se tiene una cultura política y una formación de identidad que incorpore a la inmigración (Sassen, 2014, p. 200), este país atrae trabajadores internacionales de diversos países. La presencia y tendencia de extranjeros en Japón cada ańo crece (véase gráfica 1). En 2016, el Ministerio de Justica de Japón reportaba 2307388 extranjeros, se observa pues que la migración de la población de Asia es primordial y contribuye con el $82.5 \%$ de los extranjeros en Japón. Destacan los países de China, Corea y Filipinas. En el caso del continente americano participa con el $13.2 \%$ de extranjeros, las principales naciones del 
Gráfica 1. Número de extranjeros en Japón, 2006-2016

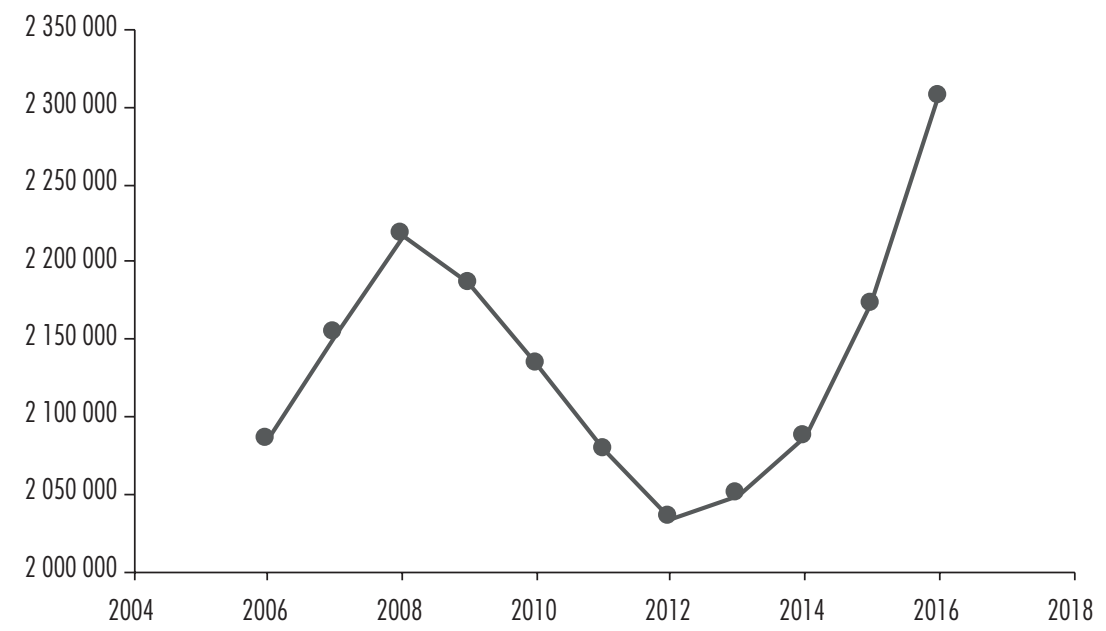

Fuente: elaboración propia, Ibídem, www.e-stat.go.jp

flujo migratorio a Japón son Brasil, Estados Unidos y Perú. Europa aporta el $3.3 \%$ y los principales países que contribuyen al flujo migratorio son Reino Unido y Francia.

De manera particular, se observa que en el ańo de 2016 residían 304865 personas en Japón, provenientes del continente americano. De ellos, el 99\% tenía su origen en 10 países diferentes. Como se aprecia en el cuadro 1, la presencia de brasileńos en Japón es notable. Brasil marca la tendencia principal del flujo migratorio de América a Japón debido a la presencia de población nikkei en Brasil; en tanto, los mexicanos ocupan la posición número ocho.

Respecto a la cantidad de mexicanos en Japón, el registro de ellos en el extranjero es una tarea gubernamental realizada por la Secretaría de Relaciones Exteriores, y para ello utiliza el Sistema de Registro para Mexicanos en el Exterior (la plataforma digital se ubica el dominio http://sirme.sre.gob.mx). A pesar de ser una forma fácil de contabilizar el número de mexicanos en el extranjero, la información es parcial y limitada en función de carácter voluntario del registro. En fecha reciente, el Instituto de Mexicanos en el Exterior (IME) ha emprendido la función de promover los vínculos de los estudiantes mexicanos que residen en el extranjero, así como apoyar a la diáspora de talentos. El Ime reporta que 12496 mexicanos vivían en Asia en el año 2015. En cambio, en 2009 había 7 233, lo cual representa un incremento de 72\%. 
Cuadro 1. Principales países de origen de extranjeros en Japón, provenientes de América, 2016

\begin{tabular}{llc}
\hline Lugar & País & Personas en Japón \\
\hline 1 & Brasil & 176284 \\
2 & Estados Unidos & 53050 \\
3 & Perú & 47670 \\
4 & Canadá & 9672 \\
5 & Bolivia & 5467 \\
6 & Argentina & 2650 \\
7 & Colombia & 2319 \\
8 & México & 2262 \\
9 & Paraguay & 1924 \\
10 & Chile & 649 \\
\hline
\end{tabular}

Fuente: elaboración propia, Ibídem, www.e-stat.go.jp

Los países más significativos con presencia de mexicanos son, en orden de volumen, China, Japón, Israel, Líbano y Emiratos Árabes Unidos. Cabe subrayar, que el $44 \%$ de ellos son estudiantes y profesionistas.

De acuerdo con los datos del Ministerio de Justicia de Japón, los residentes de origen mexicano registran una tendencia al alza desde el año 2014, y se ubica en 2262 personas en 2016. Las cifras no se incluyen a los diplomáticos ni a los mexicanos bajo el estatus de corta permanencia, sólo se contabilizan a los residentes de mediana y larga estancia (véase gráfica 2).

Con relación al caso mexicano, otra fuente, la Red de Talentos Mexicanos, capítulo Japón, que forma parte del Instituto de los Mexicanos en el Exterior de la Secretaría de Relaciones Exteriores cuenta con un registro de los mexicanos en Japón, que son considerados talentos. De acuerdo con el banco de datos del capítulo Japón que incluye una diversidad de profesiones y actividades, bajo las distintas categorías de residencia: cónyuge de japonés, residente permanente, cónyuge de residente permanente y residente fijo, se observa que el 57\% de los mexicanos en Japón están en la academia y el 34\% en empresas. Con base en el nivel de estudios, el $28 \%$ son doctores, el $38 \%$ maestros y el $34 \%$ licenciados. 
Gráfica 2. Número de residentes en Japón de origen mexicano, 2006-2016

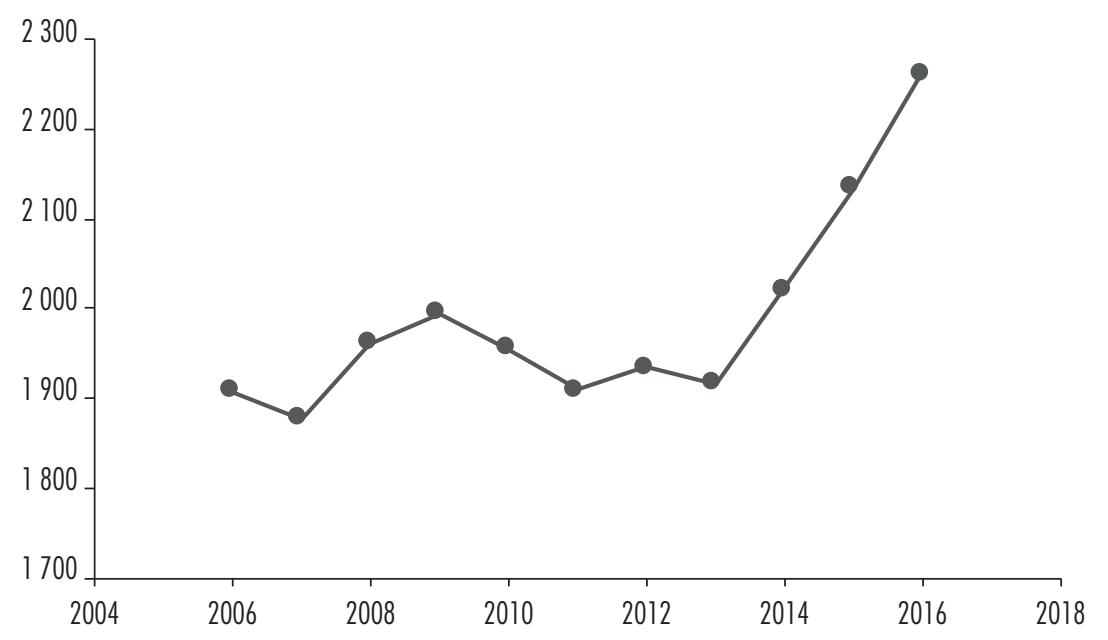

Fuente: elaboración propia, Ibídem, www.e-stat.go.jp

\section{Rasgos de la política migratoria en Japón}

El flujo migratorio está, por lo general, filtrado y regulado por las leyes de inmigración de los Estados-nación. Las leyes del sistema migratorio en Japón son diversas y complementarias, la principal es la Ley de Control de Inmigración, que establece las reglas especiales de la ley principal para los residentes permanentes. La ejecución de las leyes migratorias está a cargo del Ministerio de Asuntos Extranjeros y del Ministerio de Justicia.

Los ejes rectores de la legislación migratoria establecen que los trabajadores extranjeros deben contar con calificación y capacitación profesional para participar activamente en el mercado de trabajo y en la sociedad, con lo cual, se promueve la internacionalización del Japón. Como segundo punto, la ley establece que los trabajadores extranjeros sin calificación o baja calificación deben ser cuidadosamente monitoreados porque puede tener serios impactos en la economía y en la sociedad, específicamente afectar la vida de los ciudadanos japoneses.

En tercer eje consiste en considerar a la migración, no desde el ángulo de reposición de fuerza de trabajo por la escasez de empleados por el envejecimiento y por la disminución de la fertilidad, sino como elemento que 
favorezca la formación de una sociedad en la que las personas mayores y las mujeres puedan trabajar de manera activa.

La política migratoria es clara: son bienvenidos los profesionistas y trabajadores calificados; en tanto que está limitada la admisión de trabajadores extranjeros sin calificación, naturalmente con la excepción de la población nikkei. La política migratoria prohíbe a los migrantes acceder a empleos de baja calificación, salvo la población nikkei que puede ocupar puestos de baja calificación mediante contratación por horas. Por ejemplo, los migrantes nikkei-brasileños trabajan principalmente como operadores de máquinas en la ciudad de Ozumi al norte de Tokio en la Prefectura de Gunma en la región Kanto de Japón. En esta ocupación no se requiere formación o experiencia técnica compleja, ni habilidades de lenguaje. El salario, a finales del año de 2016 era de 1100 a 1350 yenes por hora para los hombres, y de 900 a 1000 por hora para las mujeres. Este tipo de contratación no cuenta con alguna prestación adicional. Otros lugares de marcada presencia de trabajadores nikkei son Hamamatsu, Toyota y Toyohashi.

Con la finalidad de ordenar el volumen de migrantes en Japón, se agruparon en tres categorías. El primer grupo cubre el intervalo de 140 a $220 \mathrm{mil}$ personas, y está integrado por los estudiantes internacionales, los residentes fijos y las parejas o hijos de japoneses. Como se aprecia en el cuadro 2, los estudiantes internacionales, o sea, la migración educativa son los de mayor volumen de extranjeros en Japón. Se le puede denominar la migración educacional. Muy cerca de ellos están los migrantes familiares. Actualmente, la formación de parejas de japoneses (varones y mujeres) con extranjeros ha ocasionado el fenómeno demográfico de los hijos mestizos que se denominan hafu.

El segundo grupo está integrado por los migrantes educativos temporales bajo la forma de capacitación para el trabajo. Este flujo responde a la presencia de inversiones japonesas en el mundo y a la capacitación de los empleos de las empresas multinacionales. Finalmente, en el tercer grupo se hallan la transferencia de trabajadores de las empresas multinacionales con sede en Japón. En conjunto, es claro el flujo de inmigrante en Japón, especialmente son estudiantes y trabajadores especializados. 
Cuadro 2. Extranjeros en Japón, por condición de residencia, 2010-2014

\begin{tabular}{|c|c|c|c|c|c|}
\hline Primer Grupo & 2010 & 2011 & 2012 & 2013 & 2014 \\
\hline Estudiantes & 201511 & 188605 & 180919 & 193073 & 214525 \\
\hline Long- term resident & 194602 & 177983 & 165001 & 160391 & 159596 \\
\hline Esposa o hijos de japonés & 196248 & 181617 & 162332 & 151156 & 145312 \\
\hline \multicolumn{6}{|l|}{ Segundo Grupo } \\
\hline Entrenamiento técnico 2 & 49585 & 80816 & 88196 & 93526 & 90110 \\
\hline Entrenamiento técnico 1 & 50423 & 61178 & 63281 & 61680 & 77516 \\
\hline $\begin{array}{l}\text { Humanidades y servicios } \\
\text { internacionales }\end{array}$ & 68467 & 67854 & 69721 & 72319 & 76902 \\
\hline Ingenieros & 46592 & 42634 & 42273 & 43038 & 45892 \\
\hline Trabajo especializado & 30142 & 31751 & 33863 & 33425 & 33374 \\
\hline Actividades designadas & 72374 & 22751 & 20159 & 22673 & 28001 \\
\hline \multicolumn{6}{|l|}{ Tercer Grupo } \\
\hline Transferencia intra compañías & 16410 & 14636 & 14867 & 15218 & 15378 \\
\hline $\begin{array}{l}\text { Inversionistas y gerentes } \\
\text { de negocios }\end{array}$ & 10908 & 11778 & 12609 & 13439 & 15184 \\
\hline Entretenimiento & 9247 & 6265 & 1646 & 1662 & 1967 \\
\hline Entrenamiento & 9243 & 3388 & 1804 & 1501 & 1427 \\
\hline
\end{tabular}

Fuente: elaboración propia con base en 2015 Immigration Bureau, Ministry of Justice; Japan, Tokyo.

\section{DINÁMICA DE INVESTIGACIÓN Y FORMACIÓN DE RECURSOS HUMANOS EN MÉXICO Y JAPÓN}

La investigación básica y la aplicada son reconocidas ampliamente en Japón. Los numerosos Premio Nobel recibidos por profesores japoneses en los últimos ańos da cuenta de la importancia de la investigación científica. Otro indicador del dinamismo de la actividad científica es el número de artículos publicados; por ejemplo, en 2011, los primeros cinco lugares los ocupaban: Estados Unidos, Reino Unido, Alemania, Japón y Francia. México se ubicaba en el lugar 22 (Muñiz Trejo, 2016, p. 107). 
En lo que respecta a México se caracteriza por ser un país expulsor de mano de obra no calificada, así como, calificada y de profesionistas. Las crisis cíclicas económicas y la vulnerabilidad económica han ocasionado un flujo de emigración internacional constante. México pierde recursos humanos calificados como es el caso de científicos, investigadores y académicos, en el marco de que la internacionalización ofrece mejores posibilidades de desarrollo. Según se afirma con la creación del Sistema Nacional de Investigadores se pretendía mantener y recuperar a investigadores mediante becas a cambio de que los mantuvieran una productividad. En el año de 2011, el número de investigadores ascendió a 17 637. Sin embargo, el número de investigadores en México sigue siendo bajo (González Alamilla, 2016, p. 105). En 2015, el número de investigadores nacionales ascendió a 23 302. Además, la inversión de las empresas privadas es mínima en la investigación y desarrollo.

En México, la investigación y desarrollo descansa en las universidades e instituciones de educación superior. Sin embargo, las patentes registradas en el país por empresas radicadas en México superan notablemente al número de las registradas por las universidades. Las patentes son un medio de difusión y apropiación del conocimiento, que da lugar a un mercado de conocimiento en donde interactúan los demandantes del conocimiento para la producción.

En este mercado del conocimiento, las empresas japonesas han propiciado flujos y difusión de conocimiento intraindustrial (Cohen et al., 2002).

De acuerdo con el informe de Instituto Mexicano de Propiedad Industrial, en 2012, las instituciones de educación superior mexicanas registraron 71 patentes. En cambio, las principales empresas extranjeras, radicadas en México, registraron 1369 patentes. Para ese año, las empresas extranjeras se distribuyeron por país, de manera decreciente de la siguiente manera: Estados Unidos, 716; Japón, 246; Alemania, 231; y Francia, 176. Cabe destacar que las empresas japonesas que registraron patentes en México fueron: Onic Co., NTT Docomo, Inc., Sharp Kabashiki Kaisha, Sumitomo Metal Industries Ltd. y Nippon Steel Co. (Nieto González \& García Lima, 2016, pp. 69-70). Estas dos últimas empresas se unieron en 2012.

Estas empresas japonesas surgieron y se desarrollaron durante el siglo $\mathrm{xx}$, actualmente dominan diversos sectores de la economía global. Por ejemplo, el corporativo NTT Docomo, empresa de telecomunicaciones que ha desarrollado diversas tecnologías y para el año de 2020 plantea cristalizar el proyecto 5G (la trasmisión de datos será 100 más rápida). Esta empresa tiene, a marzo de 2016, registradas 5300 patentes en Japón y 8400 patentes fuera de la nación 
asiática. ${ }^{3}$ La empresa tiene presencia económica a través de oficinas, inversiones globales y alianzas en Europa, América y Asia. De manera particular, la investigación y desarrollo (R\&D) se realiza en Yokosuka Research Park, el cual cuenta con 90 mil metros cuadrados de infraestructura.

Por su parte, la empresa Sharp Kabashiki Kaisha se dedica a la manufactura y venta de equipo de telecomunicaciones, electrodomésticos y componentes eléctricos. Al 30 de junio de 2016, en Sharp laboran 42760 empleados: 19 795 en Japón y 22965 fuera de él. El corporativo tiene 58 compañías en 24 países. En México opera bajo el nombre de Sharp Corporation México, S. A. de C.V. Los centros de investigación y desarrollo (R\&D) están en Japón y varios países.

El grupo corporativo Nippon Steel \& Sumitomo Metal (NSSMC) está integrado desde 2012 por dos empresas que tiene su origen a mediados del siglo $\mathrm{xx}$, cuando el acero fue de vital importancia para la construcción de Japón. En el corporativo laboran 13 mil trabajadores en el mundo. NSSMC tiene diversos centros de investigación. En 2014 obtuvo el Premio Innovadores Globales de Thomson Reuters debido a su volumen de patentes, a la tasa de éxito de las mismas, a la agenda o portafolio de investigación global y a la influencia de las patentes. El corporativo es líder de patentes en su sector, más de 1200 entre 2010 y 2014.

Como se aprecia, la investigación desarrollada por las empresas en Japón establece una brecha en la actividad científica aplicada a la innovación con el desarrollo de México. Esta brecha inició su ensanchamiento en el siglo xx. En esta segunda década del siglo xxi es evidente la distancia que separa a los países en el ámbito de creación y aplicación del conocimiento. La exploración de las tres empresas niponas permite calibrar el volumen de la investigación y desarrollo, así como el modelo global de creación de patentes. Esta situación favorece el flujo migratorio de trabajadores altamente calificados hacia los $R \& D$ de Japón. Así, comprender el aumento de la movilidad de recursos humanos altamente calificados lleva a a conocer la dinámica de creación y aplicación del conocimiento por parte de las empresas globales, la cual se apoya en la formación de recursos humanos en los espacios de las universidades internacionales. 


\section{La investigación como factor de atracción}

Japón se caracteriza por instrumentar una migración selectiva como es el caso de la migración calificada y de profesionistas. Por tradición se afirma que los recursos humanos altamente calificados salían del país para dirigirse a otro. Independientemente de esta idea, hoy es más compleja la movilidad de esos recursos, ya que las empresas globales rompen las fronteras nacionales, y consolidan un sistema de investigación y desarrollo en el mundo. En donde, la movilidad de esos recursos puede darse hacia cualquier país, incluyendo el nacional. Actualmente, el flujo migratorio de personas altamente calificadas se orienta hacia las empresas globales; movilidad que está fuerte y previamente tamizada por los ranking académicos, o bien con la formación de posgrado de universidades reconocidas. Así, el reclutamiento de inmigrantes calificados se realiza bajo sistemas de evaluación que incluye el ranking de investigaciones y de publicaciones, como también la formación en posgrados internacionales con presencia global. Esto constituye un mercado laboral transnacional de recursos humanos de alto nivel. Cabe señalar que el monitoreo cuantitativo de la actividad académica se realiza a través de plataformas digitales (Lupton, 2015, p. 79).

La migración de personas altamente calificadas se dirige principalmente a las instituciones de investigación. En Japón, las tareas de investigación, tanto básica como de I\&D, se realiza actualmente en tres ámbitos: los investigadores de las universidades japonesas, las industrias y los laboratorios del gobierno japonés bajo un modelo colaborativo que busca participar activamente en el mercado competitivo mundial (Wen \& Kobayashi, 2001).

El modelo colaborativo es clave en el sistema de innovación japonés. Existe un amplio consenso en Japón que la colaboración entre las universidades japonesas y la industria deben mejorarse con el fin de satisfacer las expectativas de crecimiento en el mercado competitivo en el mundo (Wen \& Kobayashi, 2001). La colaboración entre la academia y la industria es relevante durante la reciente recesión. El gobierno de Japón aprobó en abril de 1998, la "Ley de Promoción de la Transferencia de Tecnología de la Universidad a la Industria”. Se espera que las universidades contribuyan a la economía japonesa y la sociedad, principalmente, a través de la creación de nuevos negocios (Fujisue, 1998). La relación entre la universidad y la industria ha abierto un canal de difusión del conocimiento hacia las pequeñas empresas de base tecnológica en Japón, mejorando su productividad (Fukugawa, 2013; Motohashi, 2005). Desde los años ochenta se han establecido programas de vínculo de los investigadores de universidades con la industria (Hayashi, 2003). 
Una tendencia de las grandes empresas japonesa es la internacionalización de la investigación. Las compañías electrónicas más grandes en Japón desarrollan investigación en el mundo, por ejemplo: Hitachi, Toshiba, NEC, Mitsubishi, Fujitsu, Matsushita y Sony (Shirabe, Niwa, Okuda, y Ootsuji, 1997). La estructura de Grupo Fujitsu consiste en Fujitsu Laboratories (Japón) que colabora mundialmente con tres laboratorios de investigación en el extranjero: Fujitsu Laboratories of America, Fujitsu Development Center (China) Investigación y Fujitsu Laboratories de Europa. Estos centros establecen vínculos con las principales universidades e instituciones de investigación en sus respectivas áreas (Kimura, Naoi \& Nakata, 2016).

Otro caso es el proyecto de colaboración entre la empresa y el mundo académico, se da entre Toshiba, Shimizu Corporation y el Instituto Avanzado de Japón de Ciencia y Tecnología (JAIST), los que se encuentran desarrollando un innovador sistema de información sanitaria (Uchihira, Hirabayashi, Sugihara, Hiraishi \& Ikawa, 2012). Se ha propiciado la colaboración interdisciplinaria, por ejemplo, entre médicos e ingenieros (Yoda, 2016). Ante la creciente importancia reconocida a la ciencia y la tecnología se ha creado nuevas demandas en la investigación universitaria para contribuir a la solución de los problemas sociales. En Japón, la investigación está fuertemente respaldada por políticas económicas y las estrategias de crecimiento (Sakai, 2016) y por modelos de gestión del conocimiento (Sakakura \& Kobayashi, 1991).

A pesar de la tendencia de internacionalización de las tareas de I\&D, correspondiente a las empresas multinacionales, la mayor parte de las actividades de investigación se realiza en el país de origen de las empresas y, en menor medida, en centros de investigación foránea a las empresas. Esto obedece al grado de la economía de escala, a los costos de coordinación de las actividades I\&D internacional y al arraigo de las empresas de I\&D (Belderbos, Leten $\&$ Suzuki, 2013).

Un vínculo sólido entre las universidades y la industria es la figura del posdoctorado que está abierta en los centros nacionales de investigación financiados por el gobierno, los laboratorios e institutos de las universidades y los laboratorios de investigación e innovación de las grandes corporaciones empresariales de Japón. Las convocatorias están abiertas a los estudiantes internacionales. Los salarios que se ofrecen varían dependiendo del centro de investigación; por ejemplo, a finales de 2016, el salario del posdoctorado en The National Astronomical Observatory of Japan ${ }^{4}$ (NAO), es de 350 mil

4 http://www.nao.ac.jp (consultado el 10 de octubre de 2016). 
yenes mensuales (3 400 dólares); en National Institute of Information and Communications Technology (NICT), es de 430 mil yenes (4 200 dólares), ${ }^{5}$ en National Institute of Advanced Industrial Science and Technology (AIST), 470 mil yenes (4 500 dólares). ${ }^{6}$

\section{Formación de recursos humanos internacionales: la educación en Japón}

Bajo la idea de que los estudiantes internacionales son recursos potenciales de capital humano, los gobiernos nacionales han diseñado estrategias para captar los futuros trabajadores calificados. Se considera al sistema migratorio de estudiantes como la forma ad hoc para ingresar al mercado de trabajo internacional.

Quizás el primer eslabón, en la migración de recursos humanos calificados, es cursar los estudios en el sistema educativo japonés, el cual consta de diversos niveles y categorías: secundaria alta, colegios de tecnología, colegios junior, universidades, maestría y doctorado, como se muestra en la figura 1. El Ministerio de Educación, Cultura, Deporte, Ciencia y Tecnología (MEXT) se encargó de realizar las encuestas sobre el sistema educativo japonés hasta 2003. Después esa función la adquiere Japan Student Services Organization, (JASSo) establecida en el año de 2004.

El Ministerio de Justicia reporta un incremento importante en el número de estudiantes internacionales en los diversos niveles de estudio. El número de estudiantes extranjeros en Japón ascendió a 257739 en 2016; en 2015 se registraron 226 131; en 2014, 196 882; en 2013 a 178 551. El organismo público JASso reporta, que en el año de 2015 había 152 mil estudiantes inscritos en instituciones de educación superior (IES) (véase cuadro número 3). De ellos, 11 mil están inscritos en programas educacionales de corta duración, lo cual significa que están en programas de menos de seis meses con la condición de visa de College Student. El 55.8\% de los estudiantes son hombres y el $44.2 \%$ son mujeres.

http://www.nict.go.jp (consultado el 10 de octubre de 2016).

Idem. 
Figura 1. Sistema educativo japonés, 2015

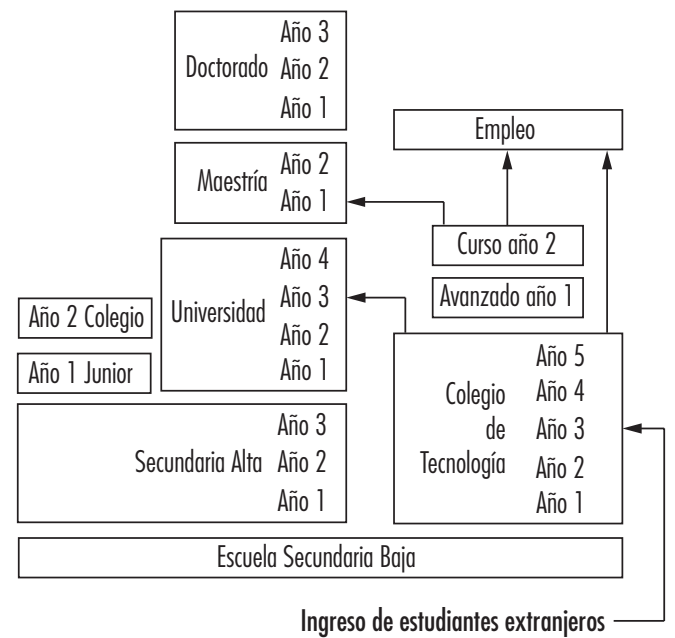

Fuente: Japan Student Service Organization, Result of an Annual Survey of International Students in Japan, 2015.

Cuadro 3. Estudiantes internacionales por tipo de institución en Japón, 2011-2015

\begin{tabular}{cccccccc}
\hline & Total & $\begin{array}{c}\text { Instituciones } \\
\text { de } \\
\text { educación } \\
\text { superior }\end{array}$ & $\begin{array}{c}\text { Universidad: } \\
\text { licenciaturas, colegio } \\
\text { universitario, colegio } \\
\text { de tecnología }\end{array}$ & Posgrados & $\begin{array}{c}\text { Institutos } \\
\text { de idioma } \\
\text { iaponés }\end{array}$ & $\begin{array}{c}\text { Escuelas } \\
\text { especializadas } \\
\text { de } \\
\text { capacitación }\end{array}$ & $\begin{array}{c}\text { Cursos de } \\
\text { preparación } \\
\text { para } \\
\text { universidad }\end{array}$ \\
\hline 2011 & 163697 & 138075 & 71244 & 39749 & 25622 & 25463 & 1619 \\
2012 & 161848 & 137756 & 71361 & 39641 & 25167 & 24092 & 1587 \\
2013 & 168145 & 135519 & 69339 & 39567 & 32626 & 24586 & 2027 \\
2014 & 184155 & 139185 & 67782 & 39979 & 44970 & 29277 & 2197 \\
2015 & 208379 & 152062 & 69405 & 41396 & 56317 & 38654 & 2607 \\
\hline
\end{tabular}

Fuente: elaboración propia con base en la gráfica 2 de Trends in Number of International Students by Institutional Type. Japan Student Service Organization, Result of an Annual Survey of International Students in Japan, 2015. A partir del año de 2011, de acuerdo con la unificación de visas, el total del número de estudiantes incluye institutos de idioma japonés. 
Antes del año de 2011, los datos se registraban de diferente forma, no obstante, se puede apreciar en la gráfica 3, que la tendencia de crecimiento del número de estudiantes extranjeros en notable desde los años ochenta. La diversidad de opciones educativas para los estudiantes internacionales es amplia.

Ahora bien, ¿qué grupos se distinguen dentro de los estudiantes extranjeros en Japón? En primer lugar, están los becarios del gobierno japonés, los cuales se definen como jóvenes que estudia en Japón bajo el programa de becas del gobierno (MEXT) establecido en 1954. En segundo lugar, están los becarios de gobiernos extranjeros, son becas obtenidas por el patrocinio del país de origen, o bien, por los fondos de ayuda japonesa para el Desarrollo de Recursos Humanos (JDs, por sus siglas en inglés), así como, la beca conjunta con el gobierno de Corea. En tercer lugar, están los estudiantes internacionales de corto plazo que son estudiantes que están recibiendo educación en Japón por un periodo de un año o menos. El propósito del estudiante no es necesariamente obtener un título, sino cubrir los requisitos para ingresar a alguna universidad japonesa, o bien, para experimentar la cultura japonesa, o para dominar el idioma.

La Ley de Reconocimiento de Refugiados y Control de Inmigración (promulgada el 5 de julio de 2009) establece que un estudiante extranjero con estatus de residencia mediante una visa de College Student está inscrito en alguna de los siete tipos de instituciones educativas: $a$ ) en las universidades japonesas, $b$ ) las escuelas de posgrado, $c$ ) colegio junior, $d$ ) colegio de tecnología, e) colegios de entrenamiento profesional, $f$ ) cursos de preparación de ingreso a universidades, y finalmente, $g$ ) institutos de enseñanza del idioma japonés.

Desde el punto de vista geográfico, Japón cuenta con ocho regiones, cada una de ellas conformada por prefecturas. En todas se cuenta con infraestructura educativa para estudiantes. La distribución de los estudiantes internacionales se da en todas las regiones. Naturalmente, se concentran en la prefectura de Kando, que comprende a la megalópolis de Tokio (véase cuadro número 4).

Los estudiantes internacionales se ubican en diversas universidades tanto privadas como públicas. En primer lugar, se encuentra las instituciones educativas ubicadas en Tokio y Osaka: Universidad de Waseda, Universtiy of Tokio, Japan University of Economics, Ritsumeikan Asia Pacific University, Kyushu University y Osaka University. Las principales áreas de estudio son ingenierías, humanidades y ciencias sociales. 
Gráfica 3. Número de estudiantes extranjeros en instituciones de educación superior en Japón

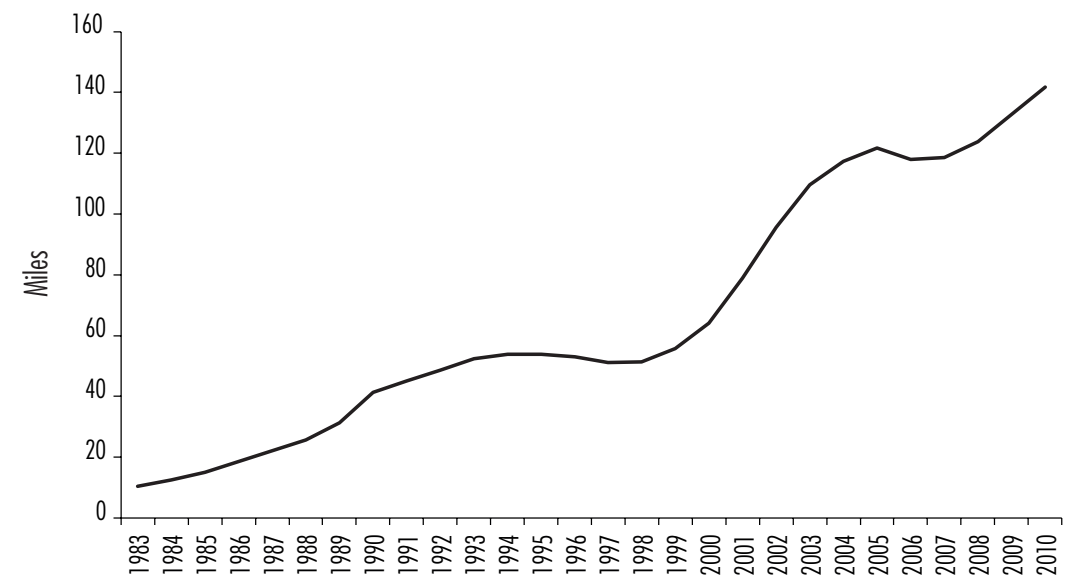

Fuente: elaboración propia con base en Japan Student Service Organization, Result of an Annual Survey of International Students in Japan, 2015.

Cuadro 4. Número de estudiantes extranieros por región en Japón

\begin{tabular}{lrr}
\hline Región & Año 2015 & Año 2014 \\
\hline Hokkaido & 2974 & 2755 \\
Tohoku & 5050 & 4328 \\
Kanto & 114778 & 97057 \\
Chubu & 17947 & 16990 \\
Kinki & 34491 & 31135 \\
Chugoku & 7881 & 7230 \\
Shikoku & 1578 & 1424 \\
Kyushu & 23680 & 23236 \\
Total & 208379 & 184155 \\
\hline
\end{tabular}

Fuente: elaboración propia con base en Japan Student Service Organization, Result of an Annual Survey of International Students in Japan, 2015. 
De acuerdo con los datos del Ministerio de Justicia de Japón, se observa una tendencia creciente de estudiantes mexicanos en Japón (véase gráfica 4); su presencia en las instituciones de educación superior y en los institutos de idiomas muestra la emergencia de un nuevo destino de formación universitaria. Las universidades japonesas han abierto algunos cursos en inglés en el marco de su internacionalización. Con este hecho, se pretende captar más estudiantes internacionales.

Cabe señalar que el flujo de estudiantes internacionales de origen mexicano está históricamente definido, tradicionalmente se dirigen hacia Reino Unido, España, Estados Unidos y Canadá debido, en entre otras razones, a la cercanía con el idioma inglés. Por otro lado, la lejanía con el idioma japonés ha sido un factor para la escasa movilidad hacia las universidades japonesas.

Cada año se suman nuevos estudiantes mexicanos en el extranjero a través del estatus de becarios. El principal grupo de estudiantes mexicanos de carácter internacional está financiado por el Consejo Nacional de Ciencia y Tecnología (Conacyt), organismo descentralizado del Estado mexicano. Según el listado de becas nuevas al extranjero por área, nivel, institución, país y género correspondientes a los años de 2014 y 2015 disponible en la plataforma datos. gob.mx, indica que en Asia se incorporaron bajo el estatus migratorio de estudiantes 31 y 30 como becarios. A Japón se dirigieron a las áreas de ingeniería, cuatro y seis, respectivamente. El mayor número de estudiantes mexicanos en Japón utilizan otros medios de financiamiento, entre ellos, los programas del gobierno japonés.

El sistema educativo en Japón está estructurado para incorporar estudiantes extranjeros en diversos momentos: en primer lugar, a los 18 años de edad el estudiante internacional puede ingresar al tercer ańo de los colegios de tecnología, al concluir el quinto año de esos colegios puede aplicar por la transferencia a la universidad, o bien, continuar con sus estudios en el nivel de cursos avanzados, después de dos años de acreditación, puede transferir a la maestría. Naturalmente, el sistema educativo establece la posibilidad de aceptar extranjeros en los niveles de licenciatura, maestría y doctorado, en las diversas universidades.

Recientemente se han abierto carreras que se imparten en inglés, aunque la mayoría de los estudios se realiza en el idioma japonés. Por lo que, la presencia de estudiantes internacionales del continente americano está representada por la comunidad nikkei que ha mantenido el contacto el idioma japonés en sus países de origen. El flujo migratorio está limitado por el dominio del idioma. Se considera que en un futuro inmediato se incorporarán más estudiantes extranjeros en virtud de la internacionalización de las universidades japonesas. 
Gráfica 4. Número de estudiantes extranjeros en Japón de origen mexicano, 2006-2016

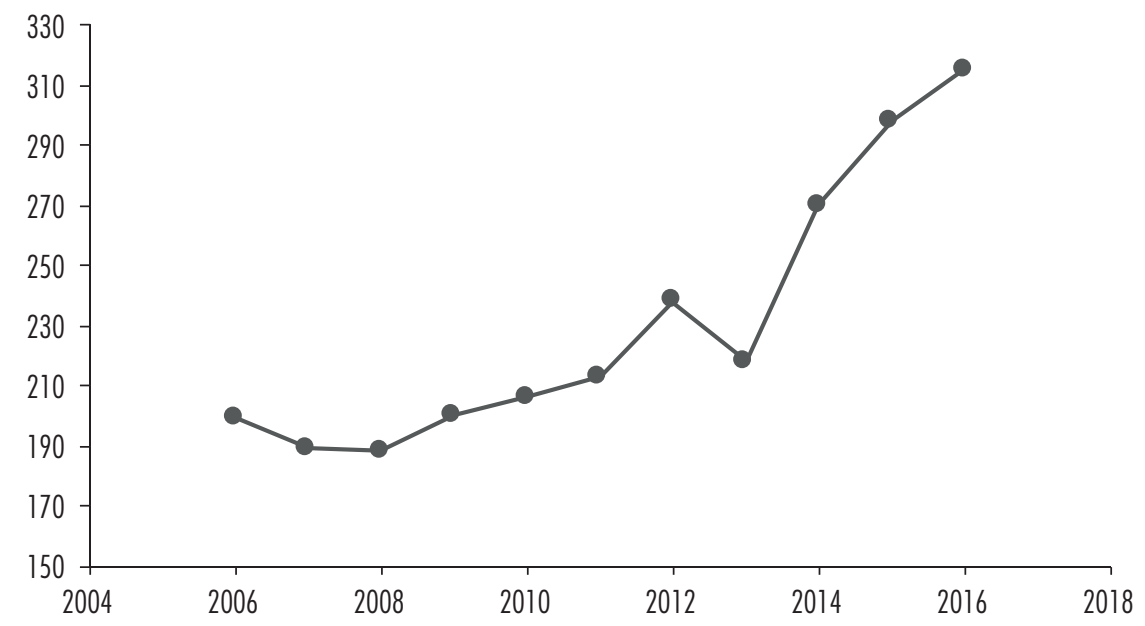

Fuente: elaboración propia, Ibídem, www.e-stat.go.jp. De 2006 a 2009 se obtuvo el total de suma de estudiantes de instituciones de educación superior (Ryugakusie) y de pre-college (Shugaku).

Cuadro 5. Número de becarios nuevos de México por continente

\begin{tabular}{lrr}
\hline Continente & 2015 & 2014 \\
\hline Europa & 1652 & 1652 \\
América & 743 & 649 \\
Oceanía & 76 & 81 \\
Asia & 30 & 31 \\
África & 1 & \\
Total & 2632 & 2515 \\
\hline
\end{tabular}

Fuente: Conacyt, archivos digitales: Becas Nuevas al Extranjero por país de destino 2015 y 2014, en <https://datos.gob.mx/busca/dataset/becasal-extranjero> 


\section{CONCLUSIONES}

En las últimas décadas se observa un aumento de inmigrantes en Japón, principalmente por el arribo de la población nikkei y por trabajadores calificados. En este artículo se abordaron los intersticios que abre la política migratoria japonesa para los recursos humanos calificados que van desde estudios en los colegios con conocimiento del idioma japonés e inglés hasta los estudios universitarios con algunas carreras en inglés. Recientemente, la Universidad de Waseda ha abierto la carrera de electrónica automotriz, autos inteligentes y robótica, la que se imparte en inglés. No obstante, el curso se realiza en su mayoría en japonés; por elcontrario, en los posgrados se privilegia el idioma inglés. A manera de conclusión se indica lo siguiente:

1) En materia de recursos altamente calificados, Japón es competitivo, según revelan los indicadores académicos: número de patentes, número de artículos, premios Nobel, número de centros de investigación y número de investigadores. Japón es un centro de atracción en la región de recursos humanos calificados, tanto en su modalidad de estudiantes internacionales como de trabajadores altamente calificados. Destaca la presencia de China, Corea y Vietnam. Los estudiantes internacionales llegan como una estrategia de formación de calidad y porque existe un flujo de migración de sus países a lo largo de la historia que ha logrado establecer redes sociales fuertes en Japón.

2) En el patrón de inmigración se observa el principio de identidad cultural, el cual ha estado presente en el flujo migratorio con el canal de la migración nikkei, que cubre trabajos calificados como no calificados. En este patrón de migración, la ciudadanía es un tema relevante, a partir del principio etnonacional.

3) La inmigración de mujeres es notoria en el ámbito de estudiantes internacionales, en el caso de trabajadoras calificadas se concentra en el ramo de "entretenimiento". Quizá sea debido a la reciente incorporación de las mujeres japonesas al mercado de trabajo, no se observa aún el fenómeno de inmigración de mujeres para realizar las tareas domésticas, como se presenta en otros países desarrollados; por ejemplo, en la ciudad-estado de Singapur y en algunos países de Europa como España son notorias la inmigración de mujeres para el cuidado doméstico.

4) El proceso de inmigración en Japón se centra en la población calificada que brinda beneficios a la economía y a la sociedad japonesa. Cabe subrayar, la presencia de recursos humanos altamente calificados que requieren los 
múltiples centros de investigación y desarrollo, localizados en todas las prefecturas de Japón.

5) En el caso de los estudiantes internacionales aún no se observa todavía la ruta de la migración hacia la obtención de la residencia permanente. Además, la inserción del egresado internacional se enfrenta al sistema de funcionamiento de la contratación de empleados, el cual tiene su propio ritmo en tiempos y formas establecidos, que se expresan en una dinámica local de contratación.

6) El sistema educativo está abierto en sus diferentes niveles para los extranjeros, desde los colegios de tecnología hasta las universidades en donde los cursos se imparten en japonés. Actualmente, algunos cursos se dictan en inglés. El sistema educativo es sumamente flexible que responde a las necesidades laborales de Japón.

7) En el contexto de la globalización, Japón ha instrumentado una política de apertura a la población extranjera como paso a la internacionalización. El patrón de inmigración cierra las puertas a la migración no calificada. Los trabajos de baja calificación continuarán realizándose por la población local. El modelo económico japonés, en general, no se soporta por la competencia salarial hacia abajo, salvo en algunos procesos productivos. Para ello, se plantea la movilidad de la comunidad nikkei, en función de la competencia de las empresas japonesas en el mundo.

8) Finalmente, la inmigración ilegal está presente, pero no forma parte de la migración de los recursos humanos calificados. Japón tiene dos tipos de respuesta: la regularización de la población ilegal o bien la deportación.

\section{BIBLIOGRAFÍA}

Bartram, D., Poros, Maritsa V. \& Monforte, P. (eds.) (2014), Key Concepts in Migration, Londres, Sage.

Bazán-Ramirez, A. \& Galván-Zariñana, G. (2013), "Incorporation of Migrant Students Returning From the United States to High Schools in Mexico", International Migration, vol. 53, núm. 1, 3-13. doi:10.111/imig. 12085

Belderbos, R., Leten, B. y Suzuki, S. (2013), "How Global is R\&D? Firmlevel Determinants of home-country bias in R\&D”, Journal of International Business Studies, vol. 44, núm. 8, 765-786. doi:10.1057/jibs.2013.33

Cohen, W. M., Goto, A., Nagata, A., Nelson, R. R., \& Walsh, J. R. (2002), "R\&D Spillovers, Patents and the Incentives to Innovate in Japan and 
the United States", Research Policy, vol. 31, núm. 8-9, 1349-1367, doi: 10.1016/s0048-7333(02)00068-9

Fujisue, K. (1998), "Promotion of Academia-industry Cooperation in Japan-establishing The Law of promoting Technology Transfer from University to Industry in Japan”, Technovation, vol. 18, núm. 6-7, 371-381. doi:10.1016/s0166-4972(98)00055-8

Fukugawa, N. (2013), "University Spillovers into Small Technology-based Firms: Channel, Mechanism, and Geography", Journal of Technology Transfer, vol. 38, núm. 4, 415-431. doi:10.1007/s10961-012-9247-x

Girón, A., Vargas, A. \& Uscanga, C. (coords.) (2015), La misión Hasekura: 400 años de su legado en las relaciones México y Japón, México, UnAM.

González Alamilla, E. (2016), "El Estado en el desarrollo científico del siglo xx", en Heriberta Castaños y Eduardo Muñiz (eds.), Mitos y realidades de la ciencia en México (epub 2016 ed., pp. 75-112), México IIEc-unAm.

Goto, J. (2008), Aging and Nikkeijin Workers in Japan: A Survey, Kobe University, Japan.

Hawthorne, L. (2014), "Indian Students and the Evolution of Study-Migration Pathway in Australia", International Migration, vol. 52, núm. 2, 3-19. doi: 10.1111 /imig. 12110

Hayashi, T. (2003), "Effect of R\&D Programmes on The Formation of University-industry-government Networks: Comparative Analysis of Japanese R\&D Programmes", Research Policy, vol. 32, núm. 8, pp. 1421-1442. doi:10.1016/s0048-7333(02)00158-0

Kimura, Y., Naoi, S. \& Nakata, T. (2016), "Research and Development and Related Activities at Fujitsu Laboratories Overseas Bases", Fujitsu Scientific \& Technical Journal, vol. 52, núm. 1, 9-18.

Lesser, J. (ed.) (2003), Searching for Home Abroad: Japanese Brazilians and Transnationalism, Estados Unidos, Duke University Press.

Lupton, D. (2015), Digital Sociology, Nueva York, Routledge.

Moskal, M. (2016), "International Students Pathways between Open and Closed Borders: Towards a Multi-scalar Approach to Educational Mobility and Labour Market Outcomes", International Migration. doi:10.1111/ imig. 12301

Motohashi, K. (2005), "University-industry Collaborations in Japan: The Role of New Technology-based Firms in transforming The National Innovation System”, Research Policy, vol. 34, núm. 5, pp. 583-594. doi:10.1016/j.respol.2005.03.001 
Muñiz Trejo, E. (2016), “Difusión y Divulgación”, en H. Castaños y E. Muñiz (eds.), Mitos y realidades de la ciencia en México (epub 2016 ed., pp. 105115), México, IIEC-UnAm.

Nieto González, Sharon, I. \& García Lima, E. (2016), "Iniciativa privada e investigación científica”, en Heriberta Castaños \& Eduardo Muñiz (eds.), Mitos y realidades de la ciencia en México (epub 2016 ed., pp. 60-70), México, IIEC-UnAM.

Sakai, H. (2016), "Why have R\&D-intensive Industries in Japan Experienced a Recent Decline in Performance? Evidence from Panel Data of Listed Firms in Japanese R\&D-intensive industries", Journal of Business Economics and Management, vol. 17, núm. 4, 527-545. doi:10.3846/16111699 .2016 .1200998

Sakakura, S., \& Kobayashi, M. (1991), "Research and Development Management in Japanese Research Institutes", Research Policy, vol. 20, núm. 6, 531-558. doi:10.1016/0048-7333(91)90045-r

Sardana, D., Zhu, Y. \& Veen, R. (2016), "Unlocking the Talents-in-Waiting: Case Study Analysis of Chinese and Indian High-skilled Migrants in South Australia", International Migration, 1-20. doi:10.1111/imig.12294

Sassen, S. (1991), The Global City: New York, London, Tokyo, Princeton, Princeton University Press.

(2014), Inmigrantes y ciudadanos: De las migraciones masivas a la Europa fortaleza (Primera reimpresión ed.), España, Siglo XXI de España Editores.

Shirabe, M., Niwa, K., Okuda, S. \& Ootsuji, H. (1997), "Survey on Tacit knowledge use in The R\&D of Japanese Companies", Innovation in Technology Management-The Key to Global Leadership: The Key to Global Leadership, 554-557. doi:10.1109/picmet.1997.653503

Tsuda, T. \& Cornelius, W. A. (2004), "Japan: Government Policy, Immigrant Reality”, en T. Tsuda, W. A. Cornelius, P. L. Martin \& J.F. Hollifield (ed.), Controlling Immigration: A Global Perspective (pp. 439-478). Stanford, California, Stanford University Press.

Uchihira, N., Hirabayashi, Y., Sugihara, T., Hiraishi, K., \& Ikawa, Y. (2012), "Knowledge Transfer in R\&D Project Management: Application to Business-academia”, Collaboration Project. Picmet '12: Proceedings-Technology Management for Emerging Technologies, 3473-3480.

Uscanga, C. (2015), "La Misión Hasekura: una valoración desde las Relaciones Internacionales", en A. Girón, A. Vargas \& C. Uscanga (coords.), La 
Misión Hasekura: 400 años de su legado en las relaciones entre México y Japón (pp. 45-57), México, UnAM.

Weiner, M. (1994), Race and Migration in Imperial Japan, Londres, Routledge.

Wen, J. \& Kobayashi, S. (2001), "Exploring Collaborative R\&D Network: some New Evidence in Japan”, Research Policy, vol. 30, núm. 8, pp. 13091319. doi:10.1016/s0048-7333(00)00152-9

Yoda, T. (2016), "The Effect of Collaborative Relationship between Medical Doctors and Engineers on The Productivity of developing Medical Devices", $R$ \& D Management, vol. 46, 193-206. doi:10.1111/radm.12131

Zapata-Barrero, R. \& Pasetti, F. (2015), "Towards a Just Mobility Regime: an Applied Ethical Approach to The Study of Migrants Admission: The Case of Skill Selection", en L.S. Talani \& S. Mchon (ed.), Handbook of the International Political Economy of Migration (pp. 74-97), Reino Unido, Edward Elgar Publishing. 